\title{
Lasing characteristics of ZnO nanosheet excited by ultraviolet laser beam
}

\section{Kota Okazaki ${ }^{\star}$, Kazuki Kubo, Tetsuya Shimogaki, Daisuke Nakamura, Mitsuhiro Higashihata,Tatsuo Okada}

Graduate School of Information Science and Electrical Engineering, Kyushu University, 744, Motooka, Nishi-ku, Fukuoka 819-0395, Japan

*Corresponding author. Tel: (+81) 92802 3681; Fax: (+81) 92802 3679; E-mail: okazaki@laserlab.ees.kyushu-u.ac.jp

Received: 26 April 2011, Revised: 22 July 2011 and Accepted: 26 July 2011

\section{ABSTRACT}

The lasing characteristics of $\mathrm{ZnO}$ nanosheets were investigated for an application to ultraviolet laser diode. ZnO nanosheets were synthesized on a silicon substrate by a CVD method, and then those $\mathrm{ZnO}$ nanosheets were examined by a photoluminescence method with a third-harmonic Nd:YAG laser (355 nm, $5 \mathrm{~ns})$. The observed emission spectra showed the obvious lasing characteristics having mode structure and a threshold for lasing. The threshold power density of a $\mathrm{ZnO}$ nanosheet was measured to be $40 \mathrm{~kW} / \mathrm{cm}^{2}$, and it would be low enough to be oscillated by an electrical pumping for ultraviolet laser diode. Copyright (C) 2011 VBRI press.

Keywords: $\mathrm{ZnO}$ nanosheet, laser; ultraviolet; photoluminescence.

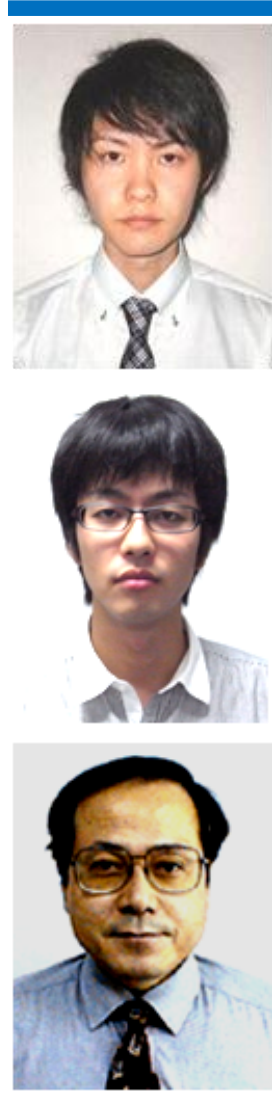

Kota Okazaki is a Ph.D. student of information science and electrical engineering (ISEE) from Kyushu University in Japan. He has been working on an evaluation of ultraviolet lasing characteristics from a single $\mathrm{ZnO}$ nanocrystal and an application to an ultraviolet laser diode.

Kazuki Kubo is a graduate student of ISEE, Kyushu University in Japan. He received his Bachelor's degree in Engineering from Kyushu University in 2010. His research interests are synthesis of $\mathrm{ZnO}$ nanowires, laser doping and laser nano-welding.

Okada Tatsuo is a professor at Department of Electrical Engineering, Kyushu University. He received a $\mathrm{Ph}$. D. degree of engineering from Kyushu University in 1981. His research interest includes development of tunable lasers, short wavelength light sources, laser processing, and spectroscopic imaging.

\section{Introduction}

Zinc oxide $(\mathrm{ZnO})$ is one of the most promising semiconductor materials in ultraviolet (UV) region. $\mathrm{ZnO}$ has a wide band-gap of approximately $3.37 \mathrm{eV}$ at room temperature and a large exciton binding energy of approximately $60 \mathrm{meV}$ that is significantly larger than the thermal energy at room temperature which corresponds to $26 \mathrm{meV}$. It can ensure an efficient exciton emission at room temperature with low excitation energy. Furthermore, since there is a great deal of resources of $\mathrm{ZnO}, \mathrm{ZnO}$ is a promising material replacing $\mathrm{GaN}$ in UV applications.

Nanostructured $\mathrm{ZnO}$ has attracted a great interest due to their importance in both scientific research and potential technological applications. From its excellent crystallization, UV stimulated emission at room temperature from optically pumped $\mathrm{ZnO}$ nanocrystals has been reported. In addition, there are various lasing mechanisms with other $\mathrm{ZnO}$ nanocrystals such as FabryPerot (FP) cavity effect in a $\mathrm{ZnO}$ nanowire[1] and a nanoribbon[2], whispering gallery mode (WGM) in a $\mathrm{ZnO}$ nanowire[3], a random lasing from $\mathrm{ZnO}$ nanoparticles[4,5] and nanobelts[6], lasing inside a $\mathrm{ZnO}$ nanosheet[7,8]. These reports show that $\mathrm{ZnO}$ nanocrystals can be candidates for excellent UV laser mediums. However, the concrete lasing mechanisms from $\mathrm{ZnO}$ nanocrystals remain to be established, and there are some major challenges to solve. For an UV LD application, it is necessary to clarify the mechanisms and the characteristics of the $\mathrm{ZnO}$ nanocrystals. In this study, we report on the further investigations of the lasing characteristics such as an oscillation spectra and a threshold power density from a single $\mathrm{ZnO}$ nanosheet under a light excitation. 


\section{Experimental}

Synthesis of ZnO nanocrystals with carbothermal CVD method

ZnO nanocrystals were synthesized by a carbothermal CVD method. The mixed sample of $\mathrm{ZnO}$ powder and Graphite was used, in which Graphite worked as a catalyst. A silicon substrate on which $\mathrm{ZnO}$ nanosheets are synthesized was used. In advance, the silicon substrate was coated by gold thin film with a thickness of $1 \mathrm{~nm}$, which was done by use of vacuum deposition equipment. The gold also works as a catalyst promoting the synthesis of $\mathrm{ZnO}$ nanocrystals. The sample was placed in an alumina boat, and then the silicon substrate was located above about $10 \mathrm{~mm}$ from the sample where the gold-coated surface was opposed to the sample. The prepared alumina boat was inserted to the center of a silica glass vacuum chamber. Next, the air in the camber was evacuated up to several Torr by a vacuum pump, and then the mixed gases of argon (Ar) and oxygen $\left(\mathrm{O}_{2}\right)$ were flowed. The amounts of gas flow of $\mathrm{Ar}$ and $\mathrm{O}_{2}$ were 100 sccm and $3 \mathrm{sccm}$, respectively.

$\mathrm{ZnO}$ nanosheets were synthesized at the temperature of $1100{ }^{\circ} \mathrm{C}$, the ambient gas pressure of 300 Torr and the synthesis time of $30 \mathrm{~min}$. Fig. 1 shows the scanning electron microscope (SEM) (KEYENCE, VE-7800S) image of the nanosheets synthesized on a silicon substrate. $\mathrm{ZnO}$ nanosheets were synthesized in a form of an island as shown in Fig. 1 (a), and the thickness of the nanosheets in Fig. 1 (b) was about $570 \mathrm{~nm}$.
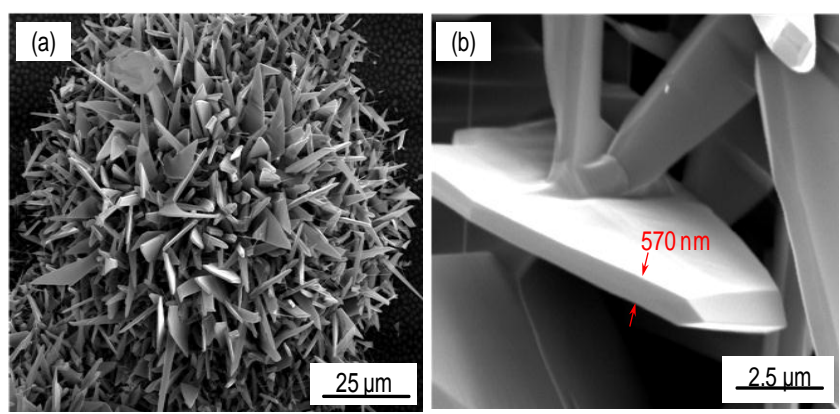

Fig. 1. SEM images of $\mathrm{ZnO}$ nanosheets synthesized on a silicon substrate by a carbothermal CVD method. The $\mathrm{ZnO}$ nanosheets form an island (a) and the width of the single nanosheet was about $570 \mathrm{~nm}(\mathbf{b})$.

\section{Arrangement for photoluminescence measurements}

The $\mathrm{ZnO}$ nanosheets were examined by a PL method. The experimental configuration for the PL method is shown in Fig. 2. A third-harmonic Nd:YAG laser which has a wavelength of $355 \mathrm{~nm}$ and a pulse duration of $5 \mathrm{~ns}$ were used as an excitation light for the $\mathrm{ZnO}$ nanosheets. In advance, $\mathrm{ZnO}$ nanosheets were dispersed on a silica glass substrate, and then the silica glass substrate was fixed on a base which could be controlled by micrometers. The $\mathrm{ZnO}$ nanosheets on the silica glass substrate were observed by a conventional optical microscope which was applied for a detail observation of the PL from a single $\mathrm{ZnO}$ nanosheet and positioned with the micrometers under the optical microscope. The excitation laser was injected through the silica glass substrate, and then the PL from a $\mathrm{ZnO}$ nanocrystal was acquired by the optical microscope and transferred to a spectrometer (Lambda Vision, TC-2000) with a light fiber.

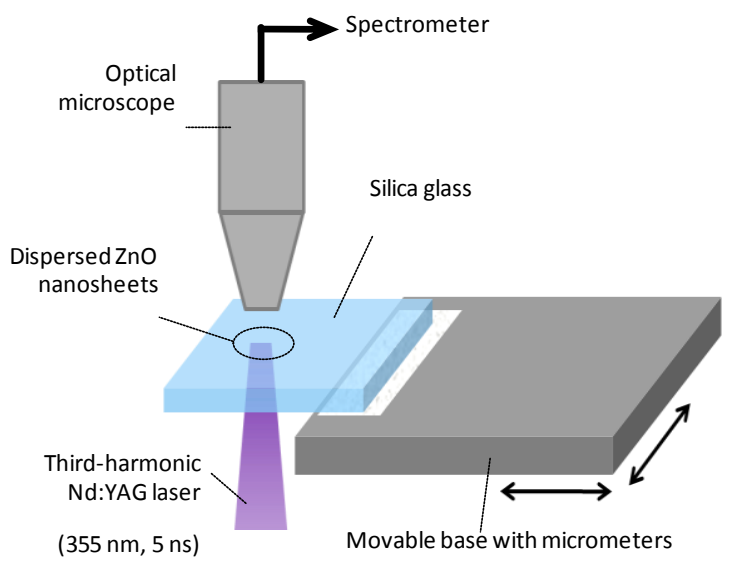

Fig. 2. Experimental configuration of photoluminescence (PL) method.

\section{Results and discussion}

The optical microscope image of the single nanosheet on a silica glass is shown in Fig. 3 (a), and the nanosheet was observed by an atomic force microscope (AFM) (KEYENCE, VN-8000M/8010M) as shown in Fig. 3 (b). The thickness of the nanosheet was about $400 \sim 900 \mathrm{~nm}$. Furthermore, the nanosheet under the excitation with the Nd:YAG laser beam is shown in Fig. 3 (c). Fig. 3 (d) shows the emission spectra from the nanosheet in changing the excitation intensities. The peak intensities at $a, b$ and $c$ of the emission spectra against the excitation power densities were shown in Fig. 3 (e). As shown in Fig. 3 (d), mode structures due to lasing from the nanosheet was observed. The lasing threshold power density was estimated to be about $40 \mathrm{~kW} / \mathrm{cm}^{2}$ from Fig. $3(\mathbf{e})$.

The pulse duration of the excitation laser was $5 \mathrm{~ns}$ which was adequately longer than the fluorescence lifetime of $\mathrm{ZnO}$ nanocrystals which is several hundreds ps and hence the excitation with a nanosecond pulse laser can be regarded as a continuous excitation. It indicates that the obtained threshold power density can be applied to an electrical pumping. Since the area of the nanosheet was estimated to be $66 \mu \mathrm{m}^{2}$ from the AFM results, threshold power was estimated to be about $26 \mathrm{~mW}$ by multiplying the area by the threshold power density. If the voltage of $30 \mathrm{~V}$ is applied to the $\mathrm{ZnO}$ nanosheet, the current of $0.88 \mathrm{~mA}$ is required to be oscillated. From these results, the laser oscillation of a $\mathrm{ZnO}$ nanosheet with electrical pumping can be realized by a total excitation from a lateral side.

Concerning $\mathrm{ZnO}$ nanosheets, there are only a few reports on lasing. The stimulated emission under the light excitation with a Ti: Sapphire laser (266 nm, $120 \mathrm{fs})$ from $\mathrm{ZnO}$ nanosheets grown by cathodic electrodeposition have been reported by F. Wang et al., and the lasing threshold was about $4.2 \mathrm{GW} / \mathrm{cm}^{2}$ [7]. In addition, E. S. Jang et al. have reported that lasing due to narrowing of PL spectra with increasing excitation power density was observed from $\mathrm{ZnO}$ nanosheets prepared by a solution process [8]. The nanosheets on Si substrate were excited by a Nd:YAG laser (355 nm, $6 \mathrm{~ns}$ ), and the lasing threshold was about 25 $\mathrm{kW} / \mathrm{cm}^{2}$. However, the oscillation mechanism whether 
WGM or random lasing was not clear because a large number of nanosheets were totally excited. B. Q. Cao et al. shows random lasing from a large number of $\mathrm{ZnO}$ nanobelts excited by third harmonic of a Q-switched $\mathrm{Nd}$ :YAG laser (355 nm, $5 \mathrm{~ns}$ ), and the lasing threshold was about $500 \mathrm{~kW} / \mathrm{cm}^{2}$ [6]. The nanobelts has similar configuration to nanosheets, even though the nanobelts are thinner $(\approx 200 \mathrm{~nm})$ than nanosheet. H. Yan et al. also shows the lasing spectra having mode-structure from a single $\mathrm{ZnO}$ nanoribbon which has a long and thin shape and the width, length and thickness were about $550 \mathrm{~nm}, 35 \mu \mathrm{m}$ and $110 \mathrm{~nm}$, respectively [2]. The nanoribbon was excited by a femtosecond laser $(310 \mathrm{~nm}, 200 \mathrm{fs})$, and the lasing threshold was $16 \mathrm{MW} / \mathrm{cm}^{2}$.
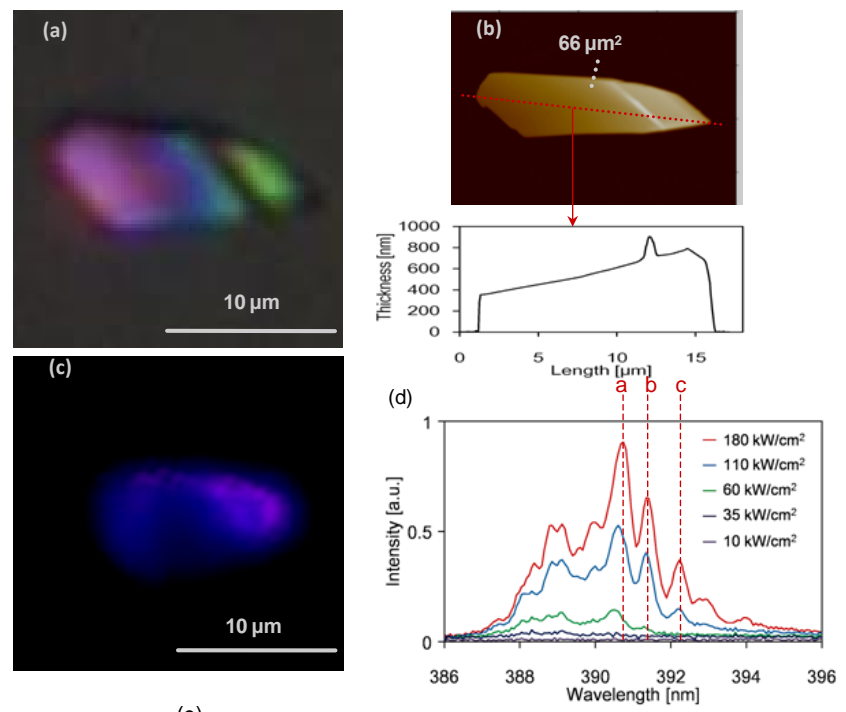

(e)

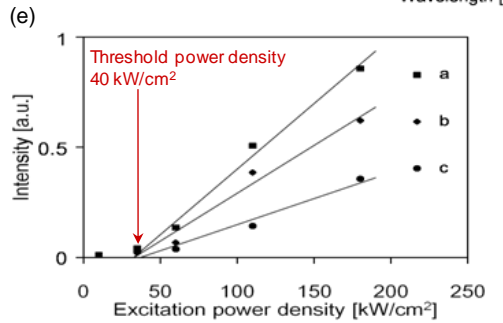

Fig. 3. (a) Optical microscope image of a single $\mathrm{ZnO}$ nanosheet on a silica glass. (b) Atomic force microscope image of the nanosheet which has the size of $400 \sim 900 \mathrm{~nm}$ in thickness and $15 \mu \mathrm{m}$ in length. (c) Optical microscope image of the nanosheet under the excitation with third-harmonic Nd:YAG laser $(355 \mathrm{~nm}, 5 \mathrm{~ns})$. (d) Emission spectra from the single $\mathrm{ZnO}$ nanosheet. The mode structures due to lasing were observed. (e) The peak intensities of the spectra plotted at a, b, and c in Fig. 3 (d) as a function of the excitation power densities. The threshold power density was estimated to be about $40 \mathrm{~kW} / \mathrm{cm}^{2}$.

In our study, the lasing from the single nanosheet prepared by a carbothermal CVD method were observed as well, and it should be noted that the oscillation spectra in Fig. 3 (d) showed obviously clear mode-structure indicating the onset of lasing due to the detailed observation from the single $\mathrm{ZnO}$ nanosheet, and the lasing threshold was about $40 \mathrm{~kW} / \mathrm{cm}^{2}$ which is considerably lower as well as the report [8] than the lasing threshold of $\mathrm{ZnO}$ nanowire which is about $200 \sim 400 \mathrm{~kW} / \mathrm{cm}^{2}[\mathbf{1 , 3 , 9}]$. The lasing spectra having mode-structure from the single $\mathrm{ZnO}$ nanosheet indicates that the lasing mechanism was attributed to micro-cavity effect including Fabry-Perot or
WGM and not random lasing. The thickness of the nanosheet was about $400 \sim 900 \mathrm{~nm}$ from the AFM result. Since the refractive index of $\mathrm{ZnO}$ is 2.4 at the wavelength around $390 \mathrm{~nm}$, the ultraviolet lights can propagate inside the nanosheet, and the total reflection angle at the boundary of $\mathrm{ZnO} /$ air is calculated to be about $25^{\circ}$ by the Snell's law, which is low enough to make the lights confinement easy. Thus, the oscillation route inside the nanosheet would not only be formed by the reflections at lateral side surfaces but also at upper and under surfaces, and WGM-like oscillation routes would be intricately formed inside the nanosheet.

\section{Conclusion}

A photoluminescence (PL) and lasing characteristics of a single zinc oxide $(\mathrm{ZnO})$ nanosheet were investigated by an optical pumping with a third-harmonic Nd:YAG laser (355 $\mathrm{nm}, 5 \mathrm{~ns}) . \mathrm{ZnO}$ nanosheets were synthesized by a carbothermal chemical vapor deposition method. According to the PL spectra, lasing from the $\mathrm{ZnO}$ nanosheet was observed, and the lasing threshold power density was about $40 \mathrm{~kW} / \mathrm{cm}^{2}$ which was considerably lower than the reported threshold of $\mathrm{ZnO}$ nanowire (200 $\left.\mathrm{kW} / \mathrm{cm}^{2} \sim\right)$. It indicates that $\mathrm{ZnO}$ nanosheets can be a superior laser medium to $\mathrm{ZnO}$ nanowires. Furthermore, the $\mathrm{ZnO}$ nanosheet can be applied to an ultraviolet laser diode because the threshold power density would be low enough to be oscillated by an electrical pumping.

\footnotetext{
Acknowledgements

The author would like to thank K. Toya for his assistance in the experiments. A part of this work was supported by a Grant-in-Aid for Scientific Research from the Japan Society for the Promotion of Science (JSPS, No. 20360142) and Special Coordination Funds for Promoting Science and Technology from Japan Science and Technology Agency is also acknowledged.
}

\section{Reference}

1. Zimmler A. M.; Capasso F.; M"uller S.; Ronning C. Semicond. Sci. Technol. 2010 25, 024001 DOI: $10.1088 / 0268-1242 / 25 / 2 / 024001$

2. Yan H.; Johnson J.; Law M.; He R.; Knutsen K.; McKinney R. J.; Pham J.; Saykally R.; Yang P. Adv. Mater. 2003 15, 1907. DOI: $10.1002 /$ adma. 200305490

3. Czekalla C.; Nobis T.; Rahm A.; Cao B.; Perez Z. J.; Sturm C.; Grund S. R.; Lorenz M.; Grundmann M. Phys. Status Solidi B 2010 $247,1282$. DOI: $10.1002 / \mathrm{pssb} .200945527$

4. Leong S. P. E.; Yu F. S. Adv. Mater. 2006 18, 1685 DOI: $10.1002 / \mathrm{adma} .200502761$

5. Leong S. P. E.; Yu F. S.; Lau P. S. Appl. Phys. Lett. 2006 89, 221109. DOI: $10.1063 / 1.2397551$

6. Cao Q. B.; Sakai K.; Nakamura D.; Palani A. I.; Gong B. H.; Xu Y. H.; Higashihata M.; Okada T. J. Phys. Chem. C 2011 115, 1702. DOI: $10.1021 / \mathrm{jp} 1097662$

7. Wang F.; Liu R.; Pan A.; Cao L.; Cheng K.; Xue B.; Wang G.; Meng Q.; Li J.; Li Q.; Wang Y.; Wang T.; Zou B. Mater. Lett. 2007, 61, 2000. DOI: 10.1016/j.matlet.2006.08.007

8. Jang S. E.; Chen X.; Won H. J.; Chung H. J.; Jang J. D.; Kim W. Y.; Choy H. J. Appl. Phys. Lett. 2010, 97, 043109. DOI: $10.1063 / 1.3466910$

9. Guo Q. R.; Nishimura J.; Matsumoto M.; Nakamura D.; Okada T. Appl. Phys. A 2008, 93, 843. DOI: $10.1007 / \mathrm{s} 00339-008-4791-9$ 


\section{AdVANCEd MATERIALS Letters}

\section{Publish your article in this journal}

ADVANCED MATERIALS Letters is an international journal published quarterly. The journal is intended to provide top-quality peer-reviewed research papers in the fascinating field of materials science particularly in the area of structure, synthesis and processing, characterization, advanced-state properties, and applications of materials. All articles are indexed on various databases including $\underline{D O A J}$ and are available for download for free. The manuscript management system is completely electronic and has fast and fair peer-review process. The journal includes review articles, research articles, notes, letter to editor and short communications. 